describing and explaining the nature and development of the diserse processes; yet the latter, which indeed includes, and is a logical extension of, the former, is by far the more important function of 'clinical chemistry' or 'chemical pethology'. In these chapters the diagnostic aids are discussed fully and extremely clearly, not as ends in themselves, nor as helping the physician to tie an appropriate label on his individual patients, but as the meens of investigating the fundamental abnormalities whether considered as a general state covered by the label or as they afflict the individual. This is an essentially modern approach, well calculated to lead to a real understanding of disease and, through that, to therapy which can be adjusted rationally to the changing needs of a particular case.

The arrangement of the book is logical in relation to its general theme and it is a remarkable tribute to contributors and editors alike that the various chapters, written by different authors and each a review capable of standing alone, cover the ground fully, connectedly, yet without unnecessary overlapping. Twenty chapters deal with the diseases of the major organs (gastrointestinal tract, liver, kidney, pancreas, major endocrine glands, muscle), with disease groups not referable to particular organs (ansemias, hypertension, etc.) and with suitably grouped metabolic disorders (diseases of nutrition, porphyrias, lipoidosis and the like). Such an arrangement, probably the best for the clinician, means that a single biochemical topic such as acid-base equilibrium may be treated in several chapters by several authors and from various points of view. This is no disadvantage but is, indeed, one of the stimulating features of the book.

One ventures to predict that "Thompson and King" will become a classic, read and studied by senior medical undergraduates, by clinicians who wish to acquire understanding of their work, and by biochemists who, professionally or through interest, are concerned in the application of their science to the study of disease.

C. P. Stewart

\section{MECHANISM IN BEHAVIOUR}

\section{Behavior Mechanisms in Monkeys}

By Heinrich Klüver. Second impression. Pp. xvii + 387. (Chicago, Ill. : University of Chicago Press ; London : Cambridge University Press, 1957.) 49s. net.

$7 \mathrm{HE}$ study of the mechanisms underlying the activity of the central nervous system has claimed the attention of workers engaged in disciplines as far removed as, for example, psychology and electronic engineering, and it is therefore not surprising that there should be marked differences of emphasis and approach. Thus, of recent years, attention has been focused on mechanisms as they exist at the level of the individual cell, on the way in which neurones influence each other's activity, and on the part played by chemical processes in the specification and patterning of cerebral structure. Concurrently, and from quite a different point of view, mathematicians and communication engineers have been prompted to suggest that many problems of cerebral organization and function can best be treated on a statistical basis and to formulate hypo. theses incorporating the established concepts of information theory and conditional probability to replace older views concerning the relationship replace older views rimulus and response. To be fruitful, such advances in knowledge and thinking must be constantly applied to what is known about the reactions of the organism as a whole, and it is therefore particularly timely that Prof. Klüver's classic monograph "Behavior Mechanisms in Monkeys", which has been out of print for many years, has now been. re-issued.

Prof. Klüver interprets the mechanism of behaviour in phenomenological terms, and his analysis is based on a study of those "general modes of reaction" from which it is possible "to predict the way an animal is going to react to a specific stimulus". He does not concern himself with the neural processes which underlie behaviour, but stresses, in his opening chapters, that little progress can be made in the investigation of cerebral function at the physiological level in the absence of firmly established experimental data concerning the overt factors governing the reactions of the individual to its environment. It is to this end that his monograph is directed.

The major portion of the book is devoted to a description and analysis of problems "selected chiefly because of their possible significance for neurophysiology". Experimental results are reported in considerdole detail and fall into two groups. The first of these deals with investigations into the features of the stimulus situation to which the monkey responds. Variations of the 'pulling-in technique', which has since been extensively applied in experimental psychology, are used for determining the equivalence or non-equivalence of stimuli in the performance of discriminations involving woight, visual appearance and sound. The findings show an incidence of response to the relational aspects of the experimental situation which is consistently high over a wide range of cues; but there are occasions, particularly in the case of some visual discriminations, in which the animal responds to absolute sensory criteria. The second group of experiments deals in a similar way with such behavioural capacitios as visual and tactual acuity, handedness and the ability to use common objects as tools.

Prof. Klüver ends with a thorough and critical analysis of his findings. He concludes that to account for the persistence of certain forms of discriminative behaviour in the face of marked changes in the environment, it is necessary to postulate the presence of some component which is identifiable throughout the range of experimental situations; but any definition of the property so abstracted as "an element which the stimuli have objectively in common" is unacceptable. Similarly, since the "response to relations" is not invariant, the unmodified concept of 'generalization' as a basic mechanism must be regarded as an over-simplification unless the individual characteristics of each stimulus situation are taken into account.

This book, which first appeared in 1933, is aimed at the serious student of behaviour rather than at the casual reader, and those unfamiliar with the extensive literature cited may have difficulty in following some of the arguments. Later developments are referred to in a new preface, but many of the original issues raised by Prof. Klüver still remain unresolved and it is gratifying that his monograph is once again readily available to a new generation of workers in the field of behavioural research.

J. T. EAYRS 